\title{
The Use of Airborne P-Band Radar Data for Land Use and Land Cover Mapping in Brazilian Amazonia ${ }^{\bullet}$
}

\author{
C. C. Freitas, S. J. S. Sant'Anna, L. S. Soler, J. R. Santos, L. V. Dutra, L. S. de Araújo, J. C. Mura, P. \\ Hernandez Filho
}

\author{
Instituto Nacional de Pesquisas Espaciais - INPE \\ Av. dos Astronautas, 1758, 12227-010 - São José dos Campos - SP, Brazil \\ Tel: +55-12-345-6475 Fax: +55-12-345-6468 \\ E-mail: \{corina,sidnei,dutra,mura\}@dpi.inpe.br, \{jroberto,lsoler,lucian,pedro\}@ltid.inpe.br
}

\begin{abstract}
The aim of this work was to analyze the potentiality of polarimetric P-band data for land use and land cover mapping in a site of the Brazilian Amazonia. These data are the first P-band image set gathered in the Brazilian Amazonia, so they represent an unique opportunity of analyzing the potentiality of this frequency for classification purposes. The stratification of land use/land cover classes was performed using a classification system specially developed for polarimetric data. Results showed that P-band data were able to discriminate forest and regeneration areas from crop, pasture and bare soil areas. Moreover, regeneration areas (older than 12 years) were successfully distinguished from primary forest and other regeneration stages.
\end{abstract}

\section{INTRODUCTION}

Microwave sensors are very important tools for monitoring tropical forest areas, due to the intense cloud cover in several regions of the Amazonian Forest. In particular, the P-band has the advantage of being more sensitive to the standing biomass variations than $\mathrm{C}$ and $\mathrm{L}$ bands, due to its longer wavelength which penetrates the forest crown, getting consequently more interaction with the tree trunks and branches [1]. The objective of this work is to analyze the potentiality of polarimetric P-band data for land use and land cover mapping in a site of the Brazilian Amazonia. It is of particular interest to know whether P-band is able to separate different stages of secondary succession, due to their role in carbon cycle.

\section{Methods}

The radar data used in this work come from a mission realized in September 2000, in the Tapajós National Forest, Brazil. The radar system used belongs to the AeroSensing Radarsysteme $\mathrm{GmbH}$, providing one look airborne polarimetric P-band slant range data, with a pixel spacing of $1.5 m \times 0.67 m$. In this work the complex images $\mathrm{HH}, \mathrm{HV}$ and $\mathrm{VV}$ were used to form a $3 \times 3$ multilook covariance matrix [2] by averaging $2 \times 5$ (range $x$ azimuth) pixels. Three elements of this matrix are intensity data and the others are complex data. The antenna pattern correction was done using a function based on homogeneous extended areas (primary forest).
The stratification of land use/land cover classes was performed with a classification system specially developed for polarimetric data $[3,4]$. This system is strongly based on statistical properties of the multivariate data, using models that stem from the multiplicative model. Three multivariate distributions are used for data modeling: the Wishart distribution (for homogeneous areas), the multivariate $\mathrm{K}$ distribution (for heterogeneous areas) and the multivariate $\mathrm{G}^{0}$ distribution (for extremely heterogeneous areas) [4]. The system uses a contextual Markovian classification technique: the Iterated Conditional Modes (ICM). The interest land use/land cover classes are bare soil (BS), crops/pasture (CP), floodplain (FP), primary forest (PF), secondary forest in four different stages of regrowth: very old (VO-older than 25 years), old (OR-between 12 and 25 years), intermediate (IRbetween 12 and 6 years) and new (NR-newer than 6 years). To train the classifier and to analyze the classification performance, several samples of the aforementioned classes were selected. This selection was done using ground truth information obtained in a field work realized during the Pband campaign. All land use classes were classified by the ICM algorithm, except the floodplain class that was extracted by a threshold procedure, in which the user could choose the value range she/he is interested in. This procedure was done because the FP class showed very high digital numbers related to other classes.

\section{III.RESULTS}

The equivalent numbers of looks $(n)$ is one of the distributions parameters used for data modeling. This parameter was estimated only once for the whole image by taking samples from homogeneous areas in amplitude data. A $\chi^{2}$ goodness of fit test for the Square Root of Gamma $\left(\Gamma^{1 / 2}\right)$ distribution was performed and the individuals $n$ were estimated from the sample moments for those areas that had a proper fit. At the end 21 samples for each amplitude data ( $\mathrm{HH}, \mathrm{HV}$ and VV) were selected and the final estimate of $n$ was computed as the mean of those individuals estimates. It is interesting to notice that about $90 \%$ of these samples were areas from primary forest and secondary succession. This might be an indication that these classes can be considered

\footnotetext{
•The authors would like to thanks CNPq (Proc. 300927/92-4, 380571/00-5, 300677/91-0), FAPESP (Proc. 00/10215-4), and FAPEMIG 00054-00.
} 
homogeneous for P-band frequency. The estimated values for each polarization were $3.417(\mathrm{HH}), 4.565(\mathrm{HV})$ and 4.146 (VV), given 4.043 as the final estimate of $n$.

Table I contains the number of training and test pixels for each class and the color legend for the classified image. Based on the training samples, a $\chi^{2}$ goodness of fit test was performed to determine the distribution that better fitted the data for each class. All classes were better fitted by the $G^{0}$ distribution, except the OR class which had the $\mathrm{K}$ distribution as the chosen one. This shows the flexibility of the $G^{0}$ distribution which has the Gamma distribution as a special case (for intensity data).

After the classification process, a Boolean operation was done to join the FP class to the classified image. By observing the confusion matrix (Table II), it can be noticed that several pixels of PF class were classified as VO class $(24.67 \%)$. The most confused classes were NR and CP. The NR class was misclassified as other regeneration types and primary forest. The $\mathrm{CP}$ class was hardly confused with $\mathrm{BS}$, showing that vegetation with structure like crops and pasture has backscatter similar to bare soil for P-band data. The OR was the best mapped class among all themes. The IR class was relatively confused with the OR, but the NR class had an expressively percent of pixels classified as IR. The FP and BS classes presented good classifications. In spite of all the mistakes, the analysis of the classified image indicates a good separation among regeneration and BS-CP classes. It can be observed that P-band data is suitable for separating old stages of regeneration (12 to 25 years) from young regeneration and from primary forest, having a better performance than $\mathrm{L}$ and $\mathrm{C}$ bands [5].

TABle I - Number Of PiXels Of Training And Test SAMPles, AND COLOR LEGEND OF CLASSIFIED IMAGE.

\begin{tabular}{cccc}
\hline Classes & Color & $\begin{array}{c}\text { Training samples } \\
\text { (pixels) }\end{array}$ & $\begin{array}{c}\text { Test samples } \\
\text { (pixels) }\end{array}$ \\
\hline PF & darkest green & 13810 & 10287 \\
VO & dark green & 35076 & 20560 \\
OR & light green & 11860 & 9641 \\
IR & lightest green & 3192 & 2980 \\
NR & yellow & 3418 & 1179 \\
BS & magenta & 18398 & 15311 \\
CP & white & 14466 & 14654 \\
FP & blue & -- & 694 \\
\hline
\end{tabular}

The estimated Kappa coefficient $(\hat{\kappa})$ was 0.5642 . However, if the interest is to separate regeneration and primary forest from recent activities areas (as for biomass studies), we may join CP and BS classes. In this case, $\hat{\kappa}$ value rises to 0.7534 .

The color composition of intensity components is presented in Fig.1(a), the classified image in Fig.1 (b) and, only for reference purposes, the Landsat-7/TM is presented in Fig.1 (c). The TM image is geo-referenced and it is not in the same projection as the radar images. In the radar composition, the recent activities appear dark, i.e., with low digital numbers. The regeneration areas occur in middle gray levels and primary forest in light gray. The floodplain are the lightest areas. It also can be seen that these areas present different texture, being the texture of primary forest regions rougher than regeneration ones.

TABLE II - CONFUSION MATRIX.

\begin{tabular}{cccccccccc}
\hline & \multicolumn{10}{c}{ Classes } & PF & VO & OR & IR & NR & BS & CP & FP & Total \\
\hline PF & 56.18 & 1.19 & 0.10 & 0.00 & 15.44 & 0.25 & 0.20 & 16.43 & 8.50 \\
VO & 24.67 & 72.99 & 3.19 & 0.00 & 1.10 & 0.00 & 0.08 & 0.43 & 23.74 \\
OR & 9.36 & 25.78 & 92.61 & 26.54 & 15.35 & 0.00 & 0.82 & 0.00 & 21.62 \\
IR & 1.38 & 0.03 & 2.93 & 62.92 & 53.44 & 0.81 & 2.22 & 0.14 & 4.50 \\
NR & 8.28 & 0.01 & 0.00 & 7.58 & 14.67 & 0.29 & 0.05 & 0.00 & 1.73 \\
BS & 0.00 & 0.00 & 0.00 & 0.91 & 0.00 & 90.30 & 80.20 & 0.00 & 34.00 \\
CP & 0.00 & 0.00 & 1.16 & 2.05 & 0.00 & 8.35 & 16.43 & 0.00 & 5.12 \\
FP & 0.13 & 0.00 & 0.00 & 0.00 & 0.00 & 0.00 & 0.00 & 83.00 & 0.78 \\
Total & 100.0 & 100.0 & 100.0 & 100.0 & 100.0 & 100.0 & 100.0 & 100.0 & 100.0 \\
\hline
\end{tabular}

A visual inspection of the classified image shows the high confusion between bare soil and crops/pasture regions. Fig. 2 to 4 highlight some areas of the classified (left), and TM (right) images of about the same region. The location of these areas are indicated in Fig. 1(b) by boxes 1 to 3, respectively.

Fig. 2 shows the good performance of the classifier in separating VO from OR classes. It can be seen from Fig. 1(c) that this VO area is very similar to primary forest in the TM image. Although the ICM classifier had a good performance in classifying the $\mathrm{VO}$ pixels, it classified many pixels of $\mathrm{PF}$ forest as belonging to $\mathrm{VO}$ (as noticed in Table II).

The top left area on Fig. 3 is an area of new regeneration that was correctly classified. The areas classified as bare soil are actually mixture areas of bare soil, pasture and crops.

The old regeneration area in Fig. 4 (light green) located at the center left, is correctly classified, as well as the neighbor area on the its right side classified as intermediate regeneration. It can be noticed that these two areas have different radiometry in the radar images, but similar radiometry in the TM image.

The box 4 in Fig. 1(b) highlights the confusion between bare soil and pasture/crops, showing that many areas of pasture and crops are classified as bare soil (also seen in the confusion matrix).

\section{Conclusions}

This paper addressed the potentiality of P-band data for classification purposes. Although the classification performance was not very good for some classes, such as for pasture/crops (which had many pixels classified as bare soil) and for new regeneration (misclassified as other types of regeneration), regeneration on advanced stages were well classified. Therefore, we may say that the polarimetric information provided by P-band data was meaningful on the discriminatory process.

\section{REFERENCES}

[1] E. Rignot, J.B. Way, C. Williams, L. Viereck, J. Yarie, "P-band radar mapping of forest biomass in boreal forests of interior Alaska," Vegetation Biomas. Pasadena, IEEE, p.1853-1855, 1994 [IGARSS'94, California].

[2] J.S. Lee, L. Du, D.L. Shuler, M.R. Grunes, "Statistical analysis and segmentation of multilook SAR imagery using partial polarimetric data," Quantitative Remote Sensing for Science and Aplications. Piscataway, IEEE, v.III, p.1422-1424, 1995 [IGARSS'95, Firenze]. 
[3] A.H. Correia, C.C. Freitas, A.C. Frery, S.J.S. Sant'Anna, "A user friendly statistical system for polarimetric SAR image classification," Revista de Teledetección, n.10, p.79-93, December 1998.

[4] A.H. Correia, "Projeto, desenvolvimento e avaliação de classificadores estatísticos pontuais e contextuais para imagens SAR polarimétricas," .Master Dissertation (in portuguese) Instituto Nacional de Pesquisas Espaciais, INPE-7178-TDI/679, São José dos Campos, SP,1998.
[5] S.J.S. Sant'Anna, C.C. Freitas, C.D. Rennó, "The use of textural features on the polarimetric SAR image classification," Image Processing Techniques, Proc. of the $2^{\text {nd }}$ Latino American Seminar on Radar Remote Sensing, Santos, São Paulo, Brazil, 11-12 Sept. 1998 (ESA SP-434).

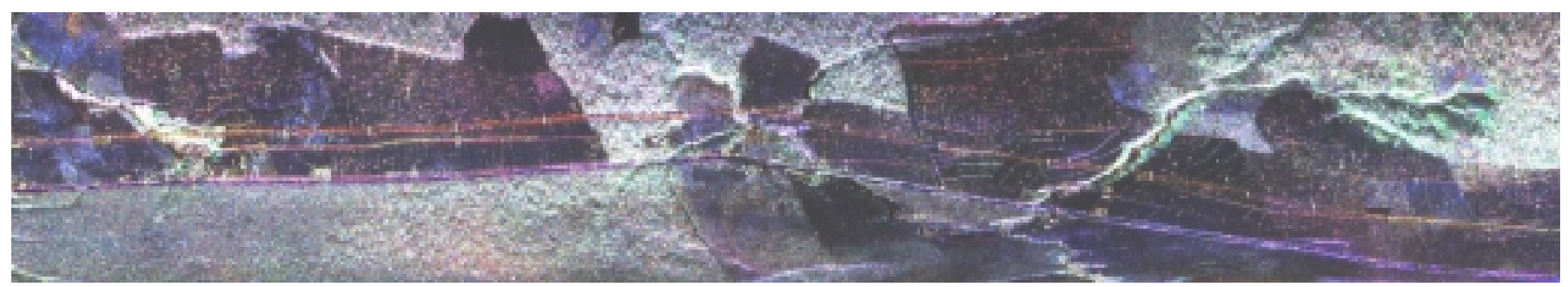

(a)

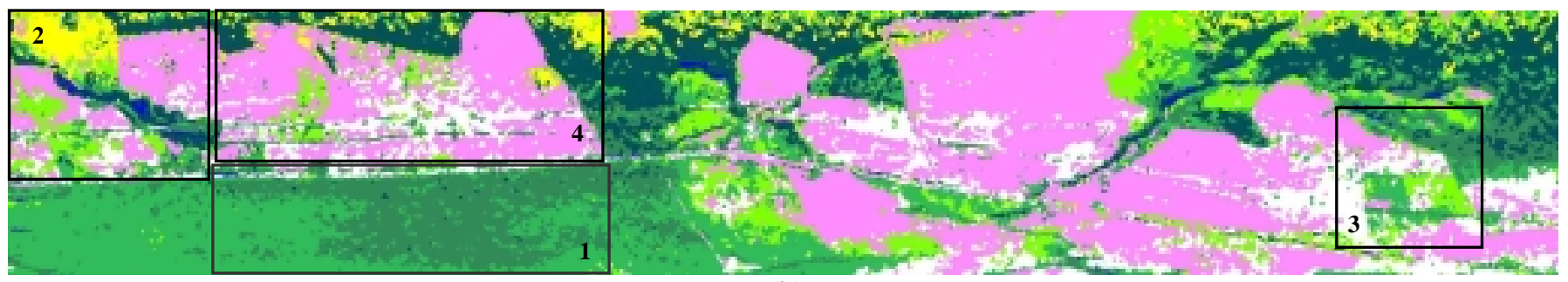

(b)

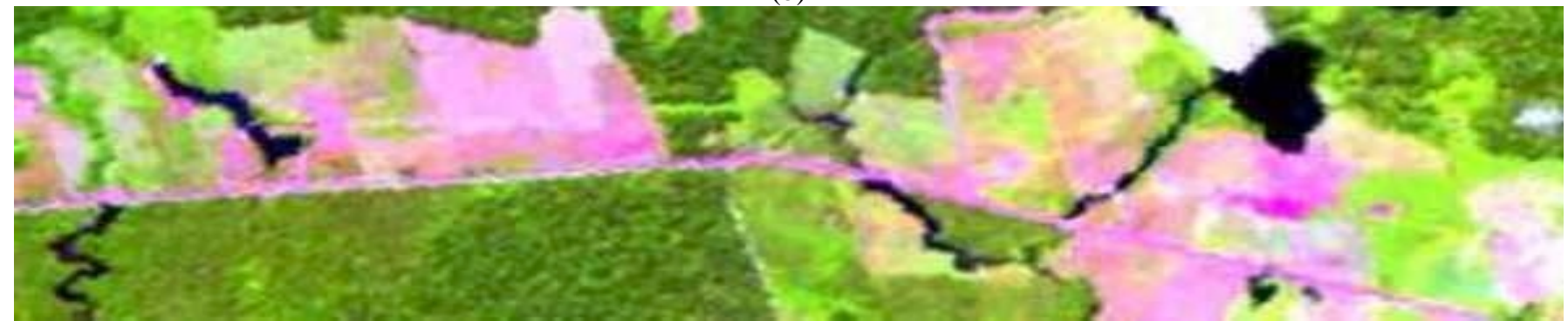

(c)

Fig.1. (a) Color composition of P-band intensity data (R-HH,G-HV,B-VV). (b) Classified image. (c) Landsat-7/TM image (R-5,G-4,B-3) 08/2000
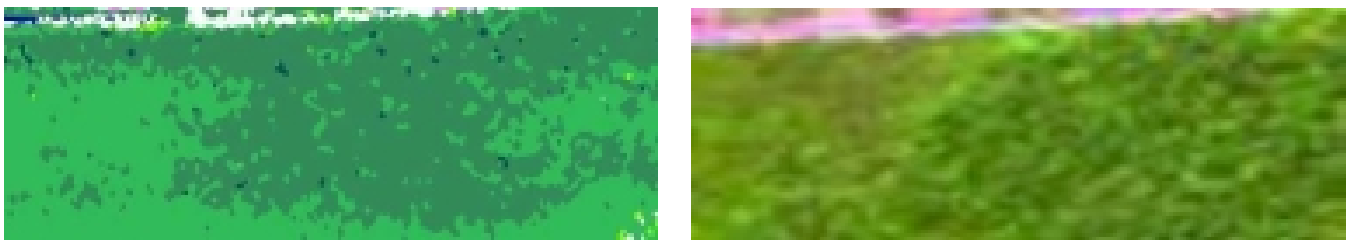

Fig.2. Zoom from area 1, illustrating the separability of very old and old regeneration areas.
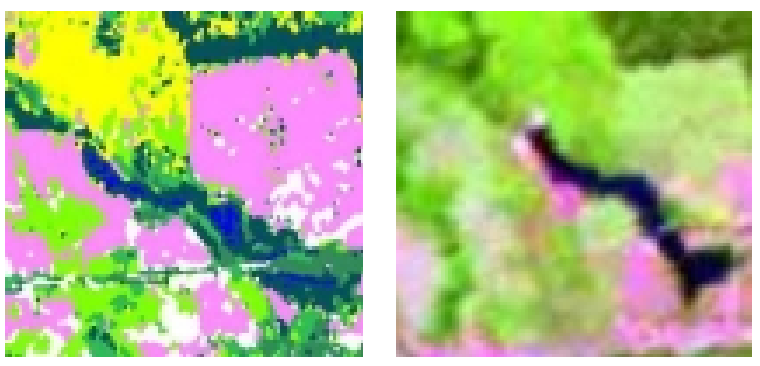

Fig.3. Zoom from area 2, illustrating the correct classification of a new regeneration area and the confusion between bare soil, pasture and crops.
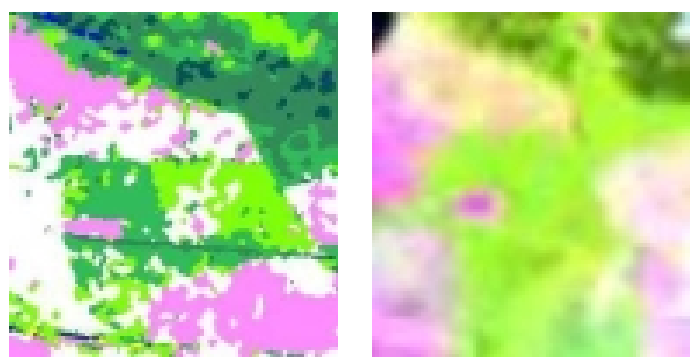

Fig.4. Zoom from area 3, illustrating the correct classification of old and intermediate regeneration areas. 